\title{
A Sparse and Condensed QP Formulation for Predictive Control of LTI Systems
}

\author{
Juan L. Jerez $^{a}$, Eric C. Kerrigan ${ }^{\mathrm{a}, \mathrm{b}}$, George A. Constantinides ${ }^{\mathrm{a}}$ \\ ${ }^{a}$ Department of Electrical and Electronic Engineering, Imperial College London, SW7 2AZ, United Kingdom \\ ${ }^{\mathrm{b}}$ Department of Aeronautics, Imperial College London, SW7 2AZ, United Kingdom
}

\begin{abstract}
The computational burden that model predictive control (MPC) imposes depends to a large extent on the way the optimal control problem is formulated as an optimization problem. We present a formulation where the input is expressed as an affine function of the state such that the closed-loop dynamics matrix becomes nilpotent. Using this approach and removing the equality constraints leads to a compact and sparse optimization problem to be solved at each sampling instant. The problem can be solved with a cost per interior-point iteration that is linear with respect to the horizon length, when this is bigger than the controllability index of the plant. The computational complexity of existing condensed approaches grow cubically with the horizon length, whereas existing non-condensed and sparse approaches also grow linearly, but with a greater proportionality constant than with the method presented here.
\end{abstract}

\section{Introduction}

In linear MPC, at every sampling instant, the optimal control input is determined through the solution of a convex optimization problem with a quadratic cost and linear constraints. MPC's natural ability for handling physical constraints has the potential to deliver significant performance benefits in many application areas. However, the very high computational demands mean that, if at all possible, expensive power-hungry hardware is often required to meet the application's sampling requirements. This has so far hindered the widespread use of the technology.

Given an estimate or measurement of the current state of the plant $\widehat{x}$, the constrained LQR problem that we will consider is

$$
\min x_{N}^{T} \widetilde{Q} x_{N}+\sum_{k=0}^{N-1}\left[\begin{array}{l}
x_{k} \\
u_{k}
\end{array}\right]^{T}\left[\begin{array}{cc}
Q & S \\
S^{T} & R
\end{array}\right]\left[\begin{array}{l}
x_{k} \\
u_{k}
\end{array}\right]
$$

\footnotetext{
* A partially modified version of this paper has been presented at CDC/ECC 2011. Corresponding author J. L. Jerez. Tel. +442072220619. This work was supported by the EPSRC (grants EP/G031576/1 and EP/I012036/1) and the EC FP7 project EMBOCON.

Email addresses: jlj05@imperial.ac.uk (Juan L. Jerez), e.kerrigan@imperial.ac.uk (Eric C. Kerrigan), gac1@imperial.ac.uk (George A. Constantinides).
}

subject to

$$
\begin{aligned}
x_{0}=\widehat{x} & \\
x_{k+1}=A x_{k}+B u_{k} & \text { for } k=0,1,2, \ldots, N-1 \\
u_{k}=K x_{k}+v_{k} & \text { for } k=0,1,2, \ldots, N-1 \\
J x_{k}+E u_{k} \leq d & \text { for } k=0,1,2, \ldots, N-1
\end{aligned}
$$

where $N$ is the horizon length, $x_{k} \in \mathbb{R}^{n}$ is the state vector at sample instant $k, u_{k} \in \mathbb{R}^{m}$ is the input vector, $(A, B)$ is controllable, $\left(Q^{\frac{1}{2}}, A\right)$ is detectable, $\left[\begin{array}{cc}Q & S \\ S^{T} & R\end{array}\right] \geq 0, R>0$ to ensure uniqueness of the solution, $\widetilde{Q} \geq 0,(2 \mathrm{c})$ represents a possible affine transformation on the input, $J \in \mathbb{R}^{l \times n}, E \in \mathbb{R}^{l \times m}, d \in \mathbb{R}^{l}$ and $l$ is the number of constraints. The techniques described in this note can easily be extended to problems with costs and constraints on the input rates, time-varying costs and constraints, as well as problems with linear terms in the cost function.

The method employed when formulating the constrained LQR problem as a quadratic program (QP) has a big impact on the problem size and structure, the resulting computational and memory requirements, as well as on the numerical conditioning. The standard approach makes use of the plant dynamics to eliminate the states from the decision variables by expressing them as an explicit function of the current state and future control 
inputs (Maciejowski, 2001). This condensed formulation leads to compact and dense QPs. In this case, the complexity of solving the QP scales cubically in the horizon length when using an interior-point method. For MPC problems that require long horizon lengths, the noncondensed formulation, which keeps the states as decision variables and considers the system dynamics implicitly by enforcing equality constraints (Rao, Wright, and Rawlings, 1998; Wright, 1993, 1996), can result in significant speed-ups. With this approach the problem becomes larger but its structure can be exploited to find a solution in time linear in the horizon length.

The non-condensed method is often also referred to as the sparse method due to the abundant structure in the resulting optimization problems. In this note, we show that this label does not provide the complete picture and that it is indeed possible to have a sparse condensed formulation that can be solved in time linear in the horizon length. In addition, we show that this method is at least as fast as the standard condensed formulation and it is faster than the non-condensed formulation for a wide variety of control problems. Our approach is based on the use of the linear feedback policy in (2c) as a mathematical trick to introduce structure into the problem. We choose $K$ such that $A+B K$ is nilpotent and show that one can formulate a QP with banded matrices in cases where the horizon length is larger than the controllability index of the plant. The use of such feedback policies for pre-stabilising predictions has been previously studied as a way of improving the conditioning of guaranteed stability MPC algorithms (Rossiter, Kouvaritakis, and Rice, 1998). However, we find it surprising that it has not yet been applied to introduce structure into the problem, as we will do here, considering the important practical implications.

Note that (2c) is effectively only a change of variables and it does not modify the optimal control problem, hence the computed optimal input is independent of the transformation used. Moreover, any procedure to guarantee stability and feasibility can still be used. For example, if the method in Scokaert and Rawlings (1998) is used, then $\widetilde{Q} \in \mathbb{R}^{n \times n}$ is the solution to the appropriate Riccati equation, which is independent of the choice of $K$ in (2c).

We start by reviewing existing QP formulations and analyzing their computational complexity in the context of primal-dual interior-point methods. However, the results stated in this paper should have a similar impact on barrier-based interior-point methods and active-set methods. We then present our sparse condensed approach and compare it with existing formulations.

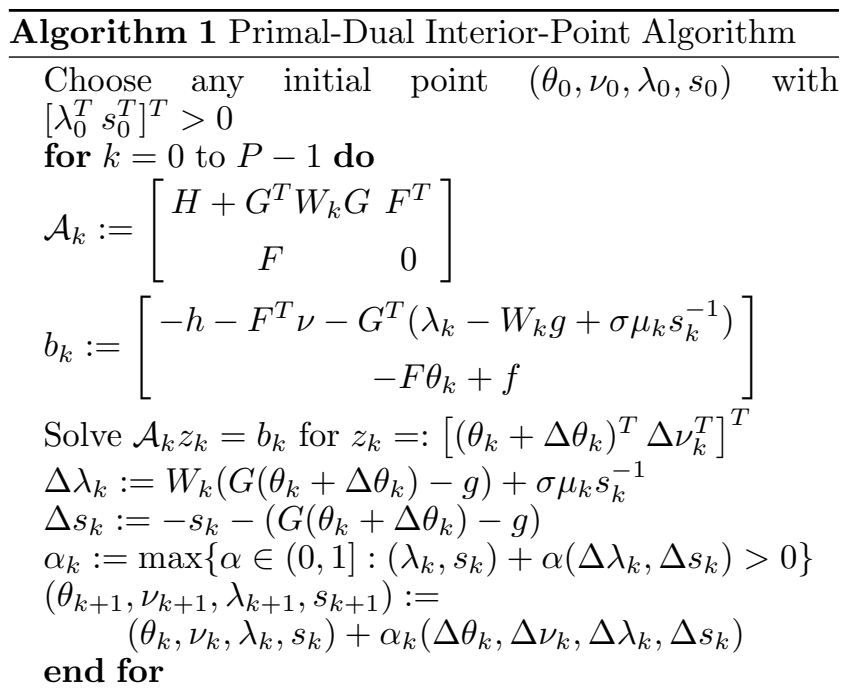

\section{QP Formulation Problem}

We consider the problem of formulating the optimal control problem (1)-(2) as a convex QP of the following form:

$$
\min _{\theta} \frac{1}{2} \theta^{T} H \theta+h^{T} \theta \quad \text { subject to } \quad F \theta=f, \quad G \theta \leq g .
$$

Primal-dual interior-point methods can be used to solve for the optimal $\theta$. Algorithm 1 is a variant of an infeasible primal-dual method (Wright, 1996), where $\nu$ and $\lambda$ are Lagrange multipliers for the equality and inequality constraints, respectively, $s$ is a vector of slack variables, $\sigma$ is a small constant between zero and one, $W_{k}:=\Lambda_{k} S_{k}^{-1}$, $\Lambda_{k}$ and $S_{k}$ are diagonal matrices containing the elements of $\lambda_{k}$ and $s_{k}$, respectively, and $\mu_{k}:=\left(\lambda_{k}^{T} s_{k}\right) /(N l)$ is a measure of sub-optimality that approaches zero at the optimum. In applications with fast dynamics, real-time requirements will impose a hard bound on the number of interior-point iterations, hence the number of interiorpoint iterations $P$ is assumed fixed a priori.

At each interior-point iteration, computing the matrix triple product $G^{T} W_{k} G$ (line 1) and solving the system of linear equations $\mathcal{A}_{k} z_{k}=b_{k}$ (line 3 ) account for most of the computation, hence we will express the overall complexity considering the cost of these operations only.

\section{Non-condensed Approach}

The future states can be kept as decision variables and the system dynamics can be incorporated into the problem by enforcing equality constraints (Rao, Wright, and Rawlings, 1998; Wright, 1993, 1996). In this case, for any arbitrary $K$, if we let $\theta:=\left[\mathbf{x}^{T} \mathbf{v}^{T}\right]^{T}$ where

$$
\mathbf{x}:=\left[\begin{array}{llll}
x_{0}^{T} & x_{1}^{T} & \ldots & x_{N}^{T}
\end{array}\right]^{T}, \quad \mathbf{v}:=\left[\begin{array}{llll}
v_{0}^{T} & v_{1}^{T} & \ldots & v_{N-1}^{T}
\end{array}\right]^{T},
$$


$h:=0$, then the remaining matrices have sparse structures that describe the control problem (1)-(2) exactly.

Assuming general constraints, the number of floating point operations (flops) for computing $G^{T} W_{k} G$ is approximately $\mathrm{Nl}(n+m)^{2}$ operations. For solving $\mathcal{A}_{k} z_{k}=$ $b_{k}$, the coefficient matrix $\mathcal{A}_{k} \in \mathbb{R}^{N(2 n+m) \times N(2 n+m)}$ is an indefinite symmetric matrix that can be made banded through appropriate row re-ordering (or interleaving of variables $\Delta \theta$ and $\Delta \nu$ ). The resulting banded matrix has a half-band of size $2 n+m$. Such a linear system can be solved using a banded $L D L^{T}$ factorization in $N(2 n+m)^{3}+4 N(2 n+m)^{2}+N(2 n+m)$ flops (Boyd and Vandenberghe, 2004, App. C), or through a block factorization method based on a sequence of Cholesky factorizations in $\mathcal{O}\left(N(n+m)^{3}\right)$ operations (Rao, Wright, and Rawlings, 1998). The memory requirements can be approximated by the cost of storing matrices $H, G, F$ and $\mathcal{A}_{k}$, which are all sparse. For time-invariant problems, these matrices mostly consist of repeated blocks.

\section{Condensed Approach}

The state variables can be eliminated from the optimization problem by expressing them as an explicit function of the current state and the controlled variables (Maciejowski, 2001):

$$
\mathbf{x}=\mathbf{A} \widehat{x}+\mathbf{B v},
$$

where $A_{K}:=A+B K$ and

$$
\mathbf{A}:=\left[\begin{array}{c}
I_{n} \\
A_{K} \\
A_{K}^{2} \\
\vdots \\
A_{K}^{N-1} \\
A_{K}^{N}
\end{array}\right], \mathbf{B}:=\left[\begin{array}{ccccc}
0 & & & & \\
B & 0 & & & \\
A_{K} B & B & \ddots & & \\
\vdots & & \ddots & & \\
A_{K}^{N-2} B & & & B & 0 \\
A_{K}^{N-1} B & A_{K}^{N-2} B & \cdots & A_{K} B & B
\end{array}\right] .
$$

In this case, if we let $\theta:=\mathbf{v}, F:=0, f:=0$, then we have an inequality constrained QP with

$$
\begin{aligned}
H:= & \mathbf{B}^{T}\left(\mathbf{Q}+\mathbf{K}^{T} \mathbf{R K}+\mathbf{S K}+\mathbf{K}^{T} \mathbf{S}^{T}\right) \mathbf{B}+ \\
& \mathbf{R}+\mathbf{B}^{T}\left(\mathbf{K}^{T} \mathbf{R}+\mathbf{S}\right)+\left(\mathbf{R K}+\mathbf{S}^{T}\right) \mathbf{B}, \\
h:= & \widehat{x}^{T} \mathbf{A}^{T}\left(\mathbf{Q B}+\mathbf{S}(\mathbf{K B}+I)+\mathbf{K}^{T}\left(\mathbf{R}(\mathbf{K B}+I)+\mathbf{S}^{T} \mathbf{B}\right)\right), \\
G:= & (\mathbf{J}+\mathbf{E K}) \mathbf{B}+\mathbf{E}, \\
g:= & \mathbf{d}-(\mathbf{J}+\mathbf{E K}) \mathbf{A} \widehat{x},
\end{aligned}
$$

where

$$
\mathbf{Q}:=\left[\begin{array}{cc}
I_{N} \otimes Q & 0 \\
0 & \widetilde{Q}
\end{array}\right], \mathbf{S}:=\left[\begin{array}{c}
I_{N} \otimes S \\
0
\end{array}\right], \mathbf{R}:=I_{N} \otimes R,
$$

$$
\mathbf{K}:=\left[I_{N} \otimes K 0\right], \mathbf{J}:=I_{N} \otimes J, \mathbf{E}:=I_{N} \otimes E,
$$

$\mathbf{d}:=1_{N} \otimes d, \otimes$ denotes a Kronecker product and $1_{N}$ denotes a vector of ones of length $N$.

When $K=0\left(u_{k}=v_{k}\right)$ or is an arbitrary stabilizing gain (Rossiter, Kouvaritakis, and Rice, 1998), $G$ is a lower block Toeplitz triangular matrix. The number of flops for computing $G^{T} W_{k} G$ can be split into $\frac{1}{2} N^{2} l m$ operations for the row update $W_{k} G$ and $\frac{1}{2} N^{3} \mathrm{~lm}^{2}$ operations for the matrix-matrix multiplication when exploiting the symmetry of the result. In terms of the system of linear equations, $\mathcal{A}_{k} \in \mathbb{R}^{N m \times N m}$ is a symmetric positive definite dense matrix, hence the problem can be solved using an unstructured Cholesky factorization in $\frac{1}{3} N^{3} m^{3}+2 N^{2} m^{2}$ flops (Boyd and Vandenberghe, 2004, App. C). The cubic growth in computational requirements with respect to the horizon length, in contrast to the linear growth exhibited by the non-condensed formulation, suggests that the non-condensed approach could be preferable for applications that require long horizons. Furthermore, memory requirements scale quadratically with $N$ because matrices are dense and there is no obviously exploitable repetition pattern.

\section{Sparse Condensed Formulation}

In this section we present a novel way to formulate the optimal control problem (1)-(2) as a structured optimization problem. We define the controllability index as the smallest number of time steps to drive the system from any $x \in \mathbb{R}^{n}$ to the origin, which is finite if the system $(A, B)$ is controllable. The nilpotency index is defined as the smallest integer $r$ such that that $A^{i}=0$ for all $i \geq r$ when $A$ is a nilpotent matrix.

Proposition 1 If the pair $(A, B)$ is controllable, we can choose $K$ such that $A_{K}$ is a nilpotent matrix with nilpotency index $r$ so that when $N>r+1$ the prediction matrix $\mathbf{B}$ in (5) is block Toeplitz, block banded lower triangular with a halfband of $(r+1) n$ elements. The last $(N-r+1) n$ rows of $\mathbf{A}$ are also zero.

PROOF. Given a reachable system $(A, B)$ there exists a feedback law such that the closed-loop dynamics matrix has arbitrary eigenvalues (Åström and Murray, 2008 ). The problem of obtaining a suitable matrix $K$ such that $A+B K$ has all eigenvalues at zero is analogous to finding a deadbeat gain in the context of static state feedback. A numerically reliable way of computing a deadbeat feedback gain in the multi-input case is not a trivial task, but the problem has been addressed by several authors (Dooren, 1984; Emami-Naeini and Franklin, 1982; Sujimoto, Inoue, and Masuda, 1993). These methods start by transforming the original system into the controllability staircase form (Dooren, EmamiNaeini, and Silverman, 1979)(ctrbf in Matlab), which 
unlike the controller canonical form, can be obtained through well-conditioned unitary transformations. The transformed system is given by

$$
\left[\begin{array}{c}
\widetilde{x}_{k+1}^{r} \\
\widetilde{x}_{k+1}^{u}
\end{array}\right]=\left[\begin{array}{cc}
A_{r} & A_{r u} \\
0 & A_{u}
\end{array}\right]\left[\begin{array}{c}
\widetilde{x}_{k}^{r} \\
\widetilde{x}_{k}^{u}
\end{array}\right]+\left[\begin{array}{c}
B_{r} \\
0
\end{array}\right] u_{k}
$$

where the subcripts $r$ and $u$ refer to the reachable and unreachable subspaces, respectively, and the matrix $A_{r}$ is in staircase form with a number of steps equal to the controllability index of the reachable subsystem $\left(A_{r}, B_{r}\right)$. These methods yield the minimum nilpotency index for $A+B K$, which is equal to the controllability index of $(A, B)$ given by

$$
r:=\left\lceil\frac{n}{\operatorname{rank}\left(B_{r}\right)}\right\rceil+r_{u}
$$

where $r_{u}$ is the nilpotency index of the unreachable subsystem $A_{u}$. The structure of $\mathbf{A}$ and $\mathbf{B}$ is clear from direct inspection of (5).

Corollary 2 If $K$ is chosen such that $A_{K}$ is nilpotent, then matrices $H$ and $G$ are banded, the size of their nonzero bands is independent of $N$, and each interior-point iteration has a complexity linear with respect to $N$.

PROOF. $X$ B yields a matrix with the same structure as $\mathbf{B}$ when $X$ is block-diagonal, and $\mathbf{B}^{T} X \mathbf{B}$ yields a symmetric banded matrix with halfband equal to the halfband of $\mathbf{B}$.

$H$ is now a block banded symmetric positive definite matrix of size $N m \times N m$ with half-band equal to $r+1$ blocks of size $m \times m$. In the time-invariant case, there are only $r+1+\frac{r(r+1)}{2}$ distinct blocks and its structure is given by

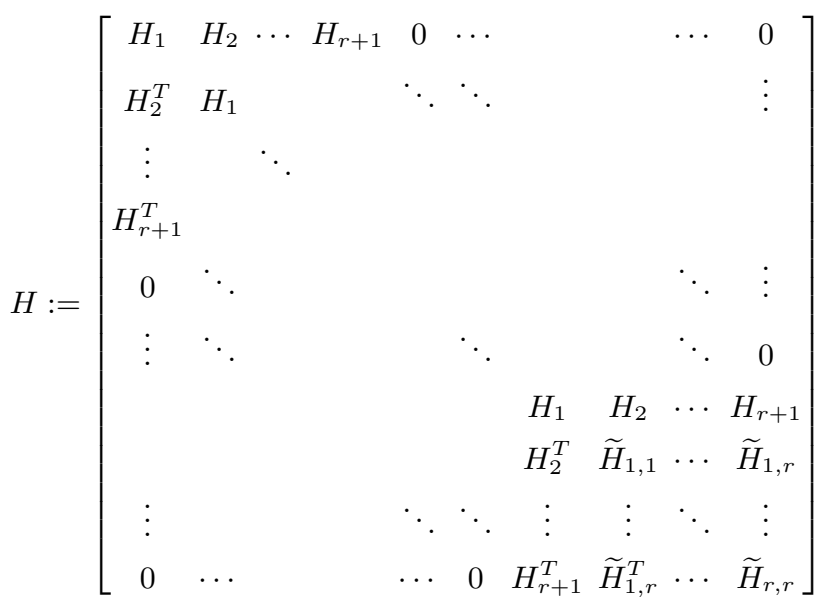

where

$$
\begin{aligned}
X:= & Q+K^{T} R K+S K+K^{T} S^{T}, \\
H_{1}:= & R+\sum_{i=1}^{r}\left(A_{K}^{i-1} B\right)^{T} X A_{K}^{i-1} B, \\
H_{j}:= & \left(A_{K}^{j-2} B\right)^{T}\left(K^{T} R+S\right)+\sum_{i=j-1}^{r-1}\left(A_{K}^{i} B\right)^{T} X A_{K}^{i-j+1} B \\
& \text { for } j=2, \ldots, r+1, \\
\widetilde{H}_{k, k}:= & R+\sum_{i=1}^{r-k}\left(A_{K}^{i-1} B\right)^{T} X A_{K}^{i-1} B+\left(A_{K}^{r-k} B\right)^{T} \widetilde{Q} A_{K}^{r-k} B \\
& \text { for } k=1, \ldots, r, \\
\widetilde{H}_{k, k+j}:= & \left(A_{K}^{j-1} B\right)^{T}\left(K^{T} R+S\right)+ \\
& \quad \sum_{i=j}^{r-k-1}\left(A_{K}^{i} B\right)^{T} X A_{K}^{i-j} B+\left(A_{K}^{r-k} B\right)^{T} \widetilde{Q} A_{K}^{r-k-j} B \\
& \text { for } j=1, \ldots, r-1 \text { and } k=1, \ldots, r-j .
\end{aligned}
$$

The situation is similar for $G$ and $\mathcal{A}_{k}$. $G$ is a block Toeplitz, block banded lower triangular matrix with a half-band of $r+1$ blocks of size $l \times m$. The number of flops for computing $G^{T} W_{k} G$ is approximately $\frac{1}{2} N m(r+1) l$ for the row update plus $\frac{1}{2} N m^{2}(r+1)^{2} l$ for the matrix multiplication. The coefficient matrix $\mathcal{A}_{k} \in \mathbf{R}^{N m \times N m}$ is now a symmetric positive definite banded matrix with the same size and structure as $H$, hence the linear system can be solved using a banded Cholesky routine with a cost of $N m^{3}(r+1)^{2}+4 N m^{2}(r+1)$ flops (Boyd and Vandenberghe, 2004). Memory requirements grow linearly with $N$ and can be reduced significantly by exploiting repetition in the time-invariant case, as above.

Table 1 compares the upper bound on the number of flops for the three different QP formulations. The expressions for the sparse condensed approach assume that $N>r+1$, otherwise the matrices are dense. As a result, the sparse condensed approach is always at least as fast as the standard condensed approach. Taking a conservative assumption for the largest possible nilpotency index $r=n$, the expressions suggest that if the number of states is larger than the number of inputs, then the formulation presented in this paper will provide an improvement over the non-condensed approach in terms of the number of flops. Both these approaches will outperform the standard condensed approach for large $N$. The assymptotic bounds from Table 1 also hold for small values of the problem parameters, as confirmed by Figure 1. The flop count of an algorithm is proportional to the computational effort required, but the computational time will largely depend on the specific implementation and computing platform. The operations to be carried out using the sparse condensed approach are all banded linear algebra for which efficient software libraries exist, hence we do not consider this to be a limiting factor. 
Table 1

Comparison of the number of flops required by the different QP formulations per interior-point iteration.

\begin{tabular}{cc}
\hline & Computation \\
\hline Condensed & $\mathcal{O}\left(N^{3} m^{2}(l+m)\right)$ \\
Non-condensed & $\mathcal{O}\left(N(m+n)^{2}(l+m+n)\right)$ \\
Sparse condensed & $\mathcal{O}\left(N m^{2} r^{2}(l+m)\right)$ \\
\hline
\end{tabular}

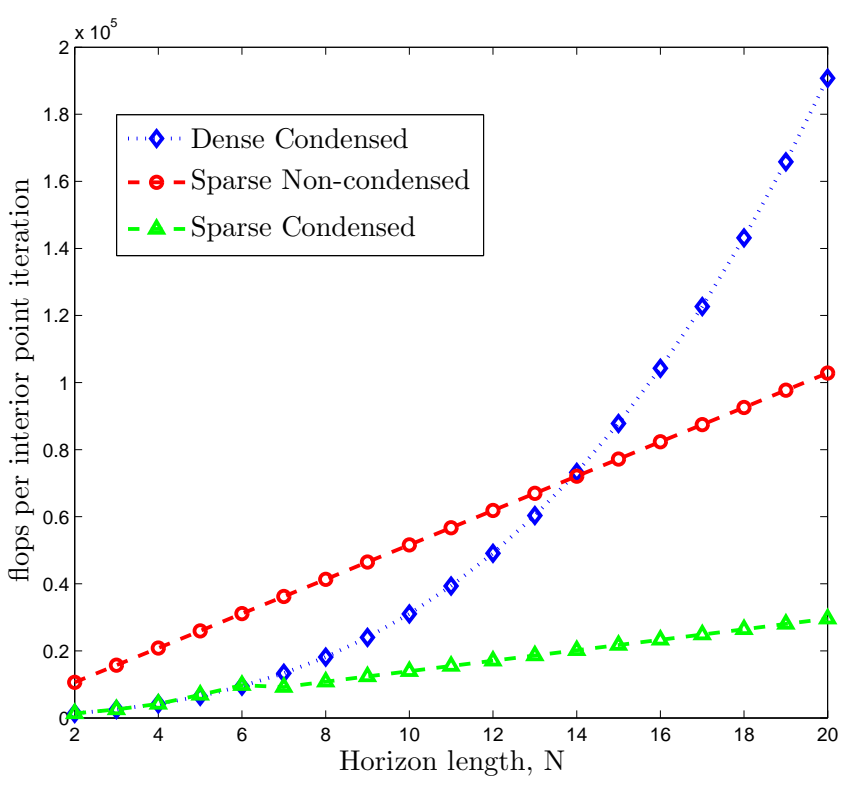

Fig. 1. Accurate count of the number of floating point operations per interior-point iteration for the different QP formulations discussed in this paper. The size of the control problem is $m=2, n=6, l=6$ and $r=3$.

Additional benefits over the non-condensed approach come from the possibility of adding input rate constraints to the optimal control problem (1)-(2) without affecting the structure of the matrices in the optimization problem (3), whereas with the non-condensed approach the inclusion of rate constraints increases the bandsize of $G$ and consequently $\mathcal{A}_{k}$. In terms of limitations, a new $K$ needs to be computed for different $(A, B)$ pairs; however, the complexity of the procedure in Sujimoto, Inoue, and Masuda (1993) is $\mathcal{O}\left(n^{2}+\operatorname{rank}\left(B_{r}\right)^{2} n\right)$, hence the approach could still be applicable to some online time-varying and nonlinear MPC applications. For LTI systems this computation is carried out offline. We have also observed that if there is a large mismatch between the sampling frequency and the plant dynamics, employing the proposed approach can lead to ill-conditioned optimization problems. However, in the absence of oversampling, the conditioning is acceptable.

\section{Conclusion}

In this note, we have presented a novel way to formulate a constrained optimal control problem as a structured optimization problem that can be solved in time lin- ear in the horizon length with an interior-point method. The structure is introduced through a suitable change of variables that results in banded prediction matrices. The proposed method has been compared against the current standard approaches and it has been shown to offer reduced computational and memory requirements for most control problems. As a result, employing the proposed approach could allow one to push the boundaries of MPC to applications where the computational burden has so far been too great, or it could allow current MPC applications to run on cheaper commodity hardware.

\section{References}

K. J. Åström and R. M. Murray. Feedback Systems: An Introduction for Scientists and Engineers. Princeton University Press, 2008.

Stephen P. Boyd and Lieven Vandenberghe. Convex Optimization. Cambridge University Press, Cambridge, UK, 2004.

Paul Van Dooren. Deadbeat control: A special inverse eigenvalue problem. BIT Numerical Mathematics, 24 (4):681-699, 1984.

Paul Van Dooren, Abbas Emami-Naeini, and L. Silverman. Stable extraction of the Kronecker structure of pencils. In Proc. 17th Conf. on Decision and Control, pages 521-524, San Diego, CA, USA, Jan 1979.

Abbas Emami-Naeini and G. F. Franklin. Deadbeat control and tracking of discrete-time systems. IEEE Transactions on Automatic Control, 27(1):176-181, Feb 1982.

Jan M. Maciejowski. Predictive Control with Constraints. Pearson Education, Harlow, UK, 2001.

Christopher V. Rao, Stephen J. Wright, and James B. Rawlings. Application of interior-point methods to model predictive control. Journal of Optimization Theory and Applications, 99(3):723-757, Dec 1998.

J. Anthony Rossiter, Basil Kouvaritakis, and Michael J. Rice. A numerically robust state-space approach to stable predictive control strategies. Automatica, 34 (1):65-73, Jan 1998.

Pierre O. Scokaert and James B. Rawlings. Constrained linear quadratic regulation. IEEE Transactions on Automatic Control, 43(8):1163-1169, Aug 1998.

Kenji Sujimoto, Akira Inoue, and Shiro Masuda. A direct computation of state deadbeat feedback gains. IEEE Transactions on Automatic Control, 38(8): 1283-1284, Aug 1993.

Stephen J. Wright. Interior-point method for optimal control of discrete-time systems. Journal on Optimization Theory and Applications, 77:161-187, 1993.

Stephen J. Wright. Applying new optimization algorithms to model predictive control. In Proc. Int. Conf. Chemical Process Control, pages 147-155, Tahoe City, CA, USA, Jan 1996. 